\title{
Riesgo preconcepcional y embarazo en la adolescencia desde un enfoque epidemiológico y preventivo
}

\section{Preconception risk and pregnancy in adolescence from an epidemiological and preventive approach}

\section{Autores:}

María Isabel García Hermida ${ }^{1,2}$

Geovanna Paola Lucero Arcos ${ }^{1,3}$

${ }^{1}$ Escuela Superior Politécnica de Chimborazo, Riobamba-Ecuador

${ }^{2}$ Centro de Salud ESPOCH Lizarzaburu, Riobamba-Ecuador

${ }^{3}$ Centro de Salud tipo B Chambo, Chambo-Ecuador

Autor de correspondencia: María Isabel García Hermida, Escuela Superior Politécnica de Chimborazo, Riobamba, Ecuador. Email: marisabelgarcia1@gmail.com. Teléfono: 0985969768 .

\section{RESUMEN}

El embarazo y el parto constituyen hechos trascendentales en la vida de la mujer; son momentos de alta carga sentimental y emocional, que implican múltiples cambios biológicos en la madre. Estos constituyen procesos que pueden estar marcados por diferentes factores de riesgo que están presentes muchas veces en la etapa preconcepcional y que se exacerban entre las embarazadas adolescentes. Al respecto se presenta una revisión bibliográfica que recoge información teórica, epidemiológica y sobre algunas formas para prevenir los respectivos factores predisponentes.

Palabras clave: embarazo en adolescencia, gestión de riesgos, promoción de la salud.

\section{ABSTRACT}

Pregnancy and childbirth are transcendental events in the life of women. They are moments of high sentimental and emotional charge, which imply multiple biological changes in the mother. These ones constitute processes that can be marked by different risk factors that are present many times in the preconceptional stage and that are exacerbated among pregnant adolescents. In this regard, a bibliographic review is presented, which gathers theoretical and epidemiological information and some ways to prevent the respective predisposing factors.

Keywords: Pregnancy in Adolescence, Risk Management, Health Promotion. 


\section{INTRODUCCIÓN}

Entre los derechos de los individuos, destaca el referente a la libre y responsable decisión acerca del número de hijos y el momento en los que tendrán; el mismo implica el deber de informarse al respecto, además de prever los recursos que se necesitarán. La pareja precisa desarrollar estrategias para controlar el número de embarazo en los niveles deseables, así como los posibles riesgos biológicos, sociales y económicos que tendrían que enfrentar en beneficio directo de la salud y bienestar familiar. ${ }^{(1)}$

El embarazo y el parto constituyen hechos trascendentales en la vida de la mujer; son momentos de alta carga sentimental y emocional, que implican múltiples cambios biológicos en ella, los mismos representan un riesgo para el organismo materno y fetal. Las probabilidades de complicaciones están presentes y se incrementan en la medida que existen determinados factores preconcepcionales que pudieran comprometer las dimensiones biopsicosociales. ${ }^{(2)}$

La morbimortalidad materna-infantil representa un problema de salud no resuelto a nivel global. La Organización Mundial de la Salud promueve la práctica de políticas nacionales dirigidas a la reducción del riesgo preconcepcional como una vía efectiva, en la disminución de los indicadores al respecto. ${ }^{(2)}$

La mortalidad materna contabiliza aquellos casos que se producen por causas relacionadas directamente o agravadas por el embarazo o su tratamiento, deben ocurrir durante la gestación, el parto o luego de los 42 días siguientes a este último. Las estadísticas mundiales indican que cada minuto tiene lugar una muerte materna; es decir, entre 500,000 y 600,000 decesos anuales relacionadas directamente con el embarazo, parto o puerperio. ${ }^{(3)}$ A nivel mundial, se calcula que existen 300 millones de mujeres adolescentes; de las cuales, aproximadamente 16 millones dan a luz cada año, representando uno por cada diez nacimientos. ${ }^{(4,5)}$

La adolescencia constituye una época de cambios significativos en el ser humano; trae variaciones físicas y emocionales; en esta, el niño comienza a tornarse en adulto, por lo que resulta definitoria para la formación y desarrollo de la personalidad, al construir su independencia rompiendo con la seguridad de su entorno infantil, pero todavía necesita apoyo familiar, escolar y social. $^{(6)}$

En países en vía del desarrollado y entre los sectores más vulnerables de la población, el embarazo adolescente constituye un importante problema para la salud pública. El riesgo resulta cuatro veces mayor en las menores de 16 años con respecto a las mayores de 20 años. ${ }^{(7)}$ Las cifras globales al respecto resultan preocupantes: cada día, 20000 mujeres menores de 18 años dan a luz en el tercer mundo; 2 de los 7,3 millones adolescentes que paren cada año son menores de 15 años. ${ }^{(8)}$ En la actualidad, este tipo de embarazos representan un problema de salud pública, debido a que son considerados de alto riesgo para el binomio madre-hijo. ${ }^{(9)}$ 


\section{DESARROLLO}

Para una adolescente, un embarazo puede tener consecuencias inmediatas y duraderas en su salud física, psicológica y social. Esta situación tiene la potencialidad de alterar toda su vida en sus dimensiones familiar, educativa y laboral, entre otras. El abandono escolar, el rechazo de sus padres, la falta de oportunidades de trabajo, el afrontamiento de la maternidad sin el apoyo de la pareja y otros constituyen algunas de las consecuencias que podrían afrontar. Al respecto, el desarrollo de programas educativos sobre salud sexual y reproductiva resultan altamente efecti$\operatorname{vos}^{(10)}$

A nivel mundial, las mujeres que viven en los países con nivel medio de desarrollo tienen 36 veces más probabilidades de riesgo de muerte por causas relacionadas con el embarazo que aquellas que habitan en los desarrollados. En estos últimos, ese indicador de salud es cada vez menor y poco frecuente, por lo que se ha constituido en un parámetro de la calidad de los servicios de salud. ${ }^{(3)}$

La prevalencia del embarazo durante esta etapa de la vida resulta alta. El 15\% de los partos registrados anualmente en Argentina involucran a madres adolescentes; fenómeno que mantiene esa tendencia por más de una década. El ejemplo claro de ese dato es que, en 2015, el total de nacimientos fue 770 040, de los cuales 111699 pertenecieron a madres adolescentes. ${ }^{(1)}$

La mortalidad materna es la consecuencia más grave del embarazo en la adolescencia; al respecto, los datos arrojan que, entre los casos de gestantes de 10 a 13 años la tasa aproximada es de 136 por cada 100000 habitantes, mientras que para las de 15 a 19 años es de 71.1 por cada 100 000 habitantes. Otros efectos negativos relacionados con ese problema de salud son: partos prolongados, abortos inseguros, incremento de la probabilidad de preeclampsia, hemorragias, infecciones de transmisión sexual, anemia, bajo peso al nacer, aumento mortalidad prenatal o durante las primeras semanas de vida del producto, entre otros. ${ }^{(7)}$

En México, el alumbramiento de madres adolescentes se considera un problema que persiste; al respecto se ha observado que, del total de las adolescentes que mantienen relaciones sexuales, aproximadamente la mitad queda embarazada; situación que se refleja en la elevación de la tasa de abortos. ${ }^{(12)}$

En Colombia, los datos de Estadísticas Vitales del Departamento Administrativo Nacional de Estadística $^{(13)}$ reportan que en 2015 se produjeron 2806 nacimientos de madres con edades comprendidas entre los 10 y 14 años, así como 63444 de adolescentes entre los 15 y 19 años. En ese mismo año, el Instituto Colombiano de Bienestar Familiar informó que ese país registra cifras de aproximadamente 408 nacimientos diarios de padres entre los 10 y 19 años. ${ }^{(14)}$

La bibliografía señala que entre el 70 y $75 \%$ de los decesos maternos ocurren durante el parto; su principal causa está dada por las hemorragias. Lamentablemente, en 2 de cada tres mujeres que sufren esta complicación no se conocen los factores de riesgo que presentan, por lo que el 
estudio epidemiológico de estos resulta de interés para la salud colectiva. La enfermedad hipertensiva del embarazo, el aborto, las infecciones de transmisión sexual entre otras patologías frecuentes durante la gravidez. ${ }^{(15)}$

El riesgo preconcepcional constituye la probabilidad que tiene una no gestante de ser dañada durante el proceso de la reproducción; está condicionado por una serie de factores, enfermedades o circunstancias únicas o asociadas que pueden repercutir desfavorablemente en el binomio, durante el embarazo, parto o puerperio. ${ }^{(16)}$

La Organización Mundial de la Salud (OMS)(17) reporta la ocurrencia 1,3 millones de muertes de adolescentes, de las cuales un alto porcentaje pudieron evitarse. En ese período y grupo poblacional, la mortalidad más elevada se observó en las edades comprendidas entre los 15 y 19 años; fenómeno que, aunque muestra un abanico multicausal, los problemas inherentes a la maternidad ocuparon el segundo lugar.

Desde la visión de desarrollo antes expuesta, Latinoamérica exhibe ejemplo de países que han implementado políticas relacionadas al alcance de una adecuada salud materna infantil. Así, Chile muestra una tasa de mortalidad materna de 18 defunciones por 100000 nacimientos; así como, una tasa infantil y perinatal de 7 a 10 por cada 1000 nacidos vivos. ${ }^{(18)}$

Esos resultados en el contexto cubano fueron obtenidos a pesar de que los estimados realizados indican que, entre 15 y $25 \%$ de su población femenina en edad fértil presentan algún factor de riesgo preconcepcional social, biológica, psíquica, ambiental o conductual. ${ }^{(19)}$

En Ecuador, 2 de cada 3 adolescentes de 15-19 años sin educación, son madres o están embarazadas por primera vez. En la última década la tendencia del incremento del embarazo en adolescentes menores de 15 años es de 74\% y en mayores de 15 años de $9 \%$, siendo la tendencia de la fecundidad adolescente, la más alta de la Región Andina, llegando a 100 por 1000 nacidos vivos. $^{(20)}$

Según el Instituto Nacional de Estadística y Censo, ${ }^{(21)}$ el embarazo en adolescentes representó el $3,4 \%$ del total de embarazos en el año 2014. La provincia del Azuay ocupo en primer lugar con un total de 4957 embarazos durante este año, en comparación con la provincia de Chimborazo que ocupó el quinto lugar, con un total 2873.

En 2017, la política del Ministerio de Salud Pública de Cuba permitió alcanzar la tasa más baja de mortalidad infantil en su historia (4,0 por 1000 nacidos vivos); lo que ubicó a ese país a la cabeza de esas cifras en Latinoamérica, además colocarse al nivel de naciones más desarrolladas en ese particular; en relación con esos datos, la mortalidad materna se redujo en 5 casos, con una tasa de 38,3 por 100000 nacidos vivos. ${ }^{(22)}$

La convergencia de factores relativos a la falta de información acerca de la sexualidad responsable, el insuficiente acceso a los métodos de anticoncepción y la persistencia de mitos y tradiciones que caracterizan el imaginario social de algunos contextos familiares, se comportan como 
elementos adversos que incrementan la vulneración de los derechos de la mujer. Lo antes dicho se ve potencializado cuando se asocia a un inadecuado abordaje en salud, debido a la presencia de barreras que limitan la adecuada organización de los servicios sanitarios. Determinante del estado de salud de la población que, al quebrantarse, impide el alcance de los principios de integralidad, equidad, accesibilidad y cobertura. ${ }^{(11)}$

Rojas Rivera plantea que ese resulta un problema de salud que puede ser mitigado mediante el trabajo salubrista en la atención primaria, a través de la intervención del médico familiar cuya labor se basa en la prevención. Este profesional está preparado para determinar los factores de riesgo individuales de cada mujer, realizar el respectivo análisis epidemiológico y un adecuado asesoramiento sobre planificación familiar y anticoncepción. ${ }^{(16)}$

El desconocimiento de los elementos relacionados con el riesgo preconcepcional favorece la ocurrencia de complicaciones en la salud para la madre y su hijo. El trabajo en la modificación de esos factores, unido al control de enfermedades presentes en la pareja durante etapas previas al embarazo, reduciría el riesgo obstétrico y perinatal. ${ }^{(16)}$

Según Jiménez González et al., las adolescentes que forman parte de procesos educativos erróneos resultan más proclives a quedar embarazadas. Esta situación se acrecienta entre los grupos sociales y étnicos históricamente menos favorecidos, con menores opciones y oportunidades en la vida, además de limitado o nulo acceso a actividades de promoción de salud sexual y reproductiva. $^{(7)}$

Numerosas experiencias en la búsqueda de la reducción del embarazo adolescente se publican en la literatura científica internacional. Muchas de las acciones que se describen están direccionadas al retraso del inicio de la vida sexual activa, la prevención de las enfermedades de transmisión sexual, el uso de métodos anticonceptivos (especialmente el preservativo), entre otras; las que, a veces, no tienen en cuenta el carácter multifactorial de este problema de salud, al no tener en cuenta determinantes sociales, educativas, económicas, psicológicas (sobre todo lo afectivo y sentimental), etc. ${ }^{(8)}$

En 2018, la mortalidad materna mostró cifras muy similares a las del año anterior. Las principales causas atribuidas a las respectivas defunciones fueron: enfermedad hipertensiva (20\%), hemorragia obstétrica $(15,56 \%)$, aborto $(15,56 \%)$ y causas indirectas $(25,56 \%) .{ }^{(23)}$

Ecuador exhibe su programa del Médico del Barrio; con el cual prevé que reducirá la mortalidad materna a nivel nacional en un $8,8 \%$; así como la tasa de mortalidad infantil en un $25,4 \%$. La labor de este profesional en la promoción de salud y prevención de enfermedades tienen en la educación de la colectividad una de sus principales herramientas. ${ }^{(24)}$

El embarazo adolescente resulta de alta preocupación para las autoridades sanitarias ecuatorianas, debido a las consecuencias económicas, sociales y psicológicas que acarrea este problema de salud que posee altos índices a nivel nacional. ${ }^{(25)}$

Lamentablemente, el ejercicio de los derechos sexuales y reproductivos por parte de los adoles- 
centes está supeditado a la vulnerabilidad social de los individuos. Jiménez-González et al. ${ }^{(7)}$ plantean que, en el contexto ecuatoriano, la violencia ha sido relacionada con el embarazo adolescente; en 2013, la Fiscalía General del Estado recibió 961 denuncias de violación de niñas menores de 14 años. Mejia et al. ${ }^{(5)}$ afirman que la violencia psicológica resulta muy común para con esas gestantes, sin menospreciar la ocurrencia de violencia física; en cualquiera de los casos, los principales agresores son frecuentemente miembros de la familia y la propia pareja.

La experimentación de la sexualidad requiere de un esfuerzo adaptativo biológico y mental; constituye parte de la asimilación del proceso de cambios que sufre el cuerpo humano en esa etapa, lo que se hace más complejo atendiendo a los cambios culturales de los adolescentes contemporáneos, que están condicionados por los medios de difusión masiva, sin la toma de conciencia de la responsabilidad que implica. ${ }^{(26)}$

Manion et al. ${ }^{(27)}$ plantean que, comúnmente, las adolescentes no se encuentran preparadas ni poseen la madurez para asumir la maternidad. En su investigación observaron que las actitudes ante la lactancia materna no era la esperada.

Bermúdez Pérez et al. ${ }^{(28)}$ establecen que el consumo de sustancias tóxicas constituye uno de los factores de riesgo preconcepcional más importante. Esos autores citan fuentes bibliográficas en las que se menciona que más del 50\% de las personas comienzan a beber alcohol antes de los 17 años y se mantienen con ese hábito durante su vida adulta.

La mayoría de las mujeres que se embarazan restan importancia a su alimentación. El correcto estado nutricional garantiza una adecuad ganancia de peso, disminuye la posibilidad de complicaciones durante la gestación y favorece la salud fetal; esas consecuencias se magnifican en el caso de madres adolescentes. ${ }^{(29)}$

Los investigadores Monterrosa Castro et al. ${ }^{(9)}$ concluyeron que las adolescentes de su muestra de estudio tenían una percepción poco definida y madura sobre el embarazo, incluso algunas jóvenes valoraron su gestación y la maternidad como una forma de cumplir uno de sus proyectos de vida.

Gálvez Henry et al. ${ }^{(30)}$ enlistan un número de consecuencias sociales que se asocian al embrazo adolescente: ${ }^{(31)}$

- Interrupción de los estudios.

- Baja capacitación para enfrentar la realidad laboral.

- Pérdida del apoyo familiar.

- Uniones inestables.

- Inmadurez para manejar la responsabilidad de madres.

Esos mismos autores destacan que la maternidad precoz puede conducir a un segundo embarazo adolescente; favorece el círculo de la pobreza en familias numerosas, incrementa el riesgo de maltrato, prostitución, alcoholismo y drogadicción. 
Restrepo Martínez et al. ${ }^{(32)}$ reconocen a la negligencia y al abuso sexual durante la infancia como importantes factores de riesgo del embarazo en la adolescencia; ellos plantean que pueden mitigarse mediante una serie de acciones educativas, legales y sociales.

La obtención de información y conocimientos sobre la prevención del embarazo por parte de los adolescentes, no garantiza su prevención; pero la educación oportuna y concreta en relación con ejercicio de la sexualidad y la vida reproductiva por parte de los diferentes actores sociales responsables, resulta determinante en el comienzo de una vida sexual responsable y en el momento adecuado; por lo que esta es una estrategia efectiva para disminuir la gestación precoz o no deseada. ${ }^{(6)}$

Esos autores definen cuatro dimensiones para el estudio del fenómeno relativo al embarazo en la adolescencia: ${ }^{(6)}$

- Cultural, tiene en cuenta tradiciones, costumbres y creencias del individuo relacionados con la sexualidad, el matrimonio y el embarazo.

- Psicológica, situación emocional, sentimental y actitudinal para afrontar la gestación.

- Socioeconómica, elementos inherentes a las relaciones humanas intra y extrafamiliares, así como la situación financiera que indique el nivel de autonomía en ese sentido.

- Educacional, tiene que ver con el nivel de escolaridad y el rol que al respecto han jugado los diferentes actores sociales.

Las adolescentes poseen necesidades particulares que pueden ser cubiertas por acciones desarrolladas en diversos espacios en los que se entregue información fidedigna y adecuada; además de la instrumentación del apoyo profesional y de pares de manera continua. ${ }^{(33)}$

Vicente Peña, Peraza Rodríguez y Sánchez Nuñez ${ }^{(34)}$ definen salud reproductiva como el estado de bienestar físico, mental y social en relación con el proceso de la reproducción. La forma en que se aplique el concepto de riesgo reproductivo constituye la herramienta fundamental para establecer dicho estado; este enfoque parte de factores preconcepcionales, obstétricos, perinatales e infantiles.

La magnitud de la vulnerabilidad al daño reproductivo depende de la existencia de determinadas características biopsicosociales y ambientales, que algunos autores definen como factores de riesgo preconcepcional. ${ }^{(1)}$

Ese posicionamiento con un enfoque multifactorial fue confirmado por Duque et al. ${ }^{(35)}$ Durante su proceso investigativo observaron que los padres con mayor número de hijos que presentaban cardiopatías, también tenían diferentes limitaciones socioeconómicas y educativas; además de asociarse significativamente con factores de riesgo ambientales y con el consumo de algún tipo de sustancias psicoactivas o medicamentos. 
En 2013, Lugones-Botell estableció una serie de factores de riesgo preconcepcional:(36)

- Multiparidad.

- Obesidad.

- Edad mayor de 35 años.

- Antecedentes de padecer anemia.

- Fibroma uterino.

- Enfermedades hematológicas.

- Gestorragias de la segunda mitad del embarazo.

- Macrosomía fetal.

- Polihidramnio.

- Embarazo múltiple.

- Eclampsia.

- Entre otros.

Resultados de algunas investigaciones apuntan hacia los factores biológicos los que con mayor frecuencia están presentes en las poblaciones de estudio, tales como: hipertensión arterial, desnutrición y determinados antecedentes obstétricos desfavorables. Al respecto, los equipos básicos de salud deben establecer estrategias dirigidas hacia su identificación y control. ${ }^{(35,37)}$

Nápoles Méndez ${ }^{(22)}$ hace referencia a que, en Cuba, las causas directas más frecuentes de muerte materna fueron: fenómenos embólicos, hemorragias relacionadas con el parto y trastornos hipertensivos; mientras que entre las indirectas destacaron: enfermedades del sistema circulatorio, de la sangre y del sistema digestivo.

Otro elemento que requiere especial atención es el riesgo obstétrico; el que se define como la suma los factores intrínsecos a la mujer, con la potencialidad para generar complicaciones en el embarazo, parto, puerperio. ${ }^{(38)}$ La valoración de este clasifica la gestación como: normal, de bajo riesgo o de alto riesgo.

Vigil-Iglesias et al. ${ }^{(38)}$ establecen los siguientes factores de riesgo obstétrico:

- Embarazo adolescente, incrementa la probabilidad de ocurrir preeclampsia-eclampsia, prematurez y el bajo peso al nacer.

- Embarazo en mujeres mayores de 34 años, se asocia con complicaciones por preeclampsia-eclampsia, inserción baja de placenta y atonía uterina postparto.

- Período intergenésico menor de 2 años, mayores posibilidades de presentar prematurez y restricción del crecimiento intrauterino.

- Multípara, aumento de la probabilidad de inserción baja de placenta y atonía uterina postparto. 
- Antecedente de abortos, eleva la posibilidad de repetición del evento.

- Preeclampsia-eclampsia, ídem al caso anterior.

- Hemorragia durante la segunda mitad de la gestación, ídem al caso anterior.

- Cesárea previa, se asocia con el incremento del riesgo de: inserción baja de placenta, acretismo placentario y ruptura uterina durante la labor de parto. Este se acrecienta exponencialmente en la medida aumenta el número de cesáreas previas.

- Antecedente de malformaciones congénitas en la pareja, mayor posibilidad de que se presenten en el producto.

- Antecedente de muerte fetal, se asocia con el incremento de la probabilidad de que se repita, generalmente es causada por una patología crónica de base.

- Prematurez, la que tiene alta probabilidad de repetirse en diferentes embarazos.

- Hipertensión arterial, aumenta el riesgo de preeclampsia-eclampsia, restricción del crecimiento intrauterino, además de la muerte fetal y materna en los casos más graves.

- Diabetes mellitus, se asocia con posible ocurrencia de preeclampsia-eclampsia, aborto, restricción del crecimiento intrauterino y la macrosomía fetal.

- Cardiopatía congénita o adquirida, predispone para insuficiencia cardiaca materna durante la 2a mitad del embarazo, restricción del crecimiento intrauterino, preeclampsia-eclampsia y muerte fetal. En la bibliografía le asocian con el deterioro de la salud para la madre, el feto o el recién nacido.

Duque et al. ${ }^{(35)}$ señalan que todos los profesionales de la medicina deben estar preparados para realizar acciones de orientación sobre planificación familiar. Lugones Botell(2) recomienda la realización de las siguientes estrategias con vista a reducir la morbimortalidad materno-infantil; la que resulta consecuencia de factores de riesgo existentes desde antes del embarazo:

- Priorizar la prevención de los elementos predisponentes de la morbilidad y mortalidad materno-infantil como política relacionada con la salud reproductiva.

- Controlar los factores de riesgo preconcepcional desde los niveles primarios de atención de salud, estableciendo como prioridad los elementos inherentes a la planificación familiar.

- Trabajar sistemáticamente en el diagnóstico de la morbilidad de las mujeres en edad fértil, desde las áreas del nivel de atención primaria.

- Realizar actividades de promoción y prevención en salud para reducir la ocurrencia de complicaciones durante el embarazo y el parto.

Al respecto, Nápoles Méndez ${ }^{(22)}$ propone, además, el fortalecimiento de ese control mediante la prevención, el diagnóstico oportuno y el manejo de las enfermedades genéticas.

El riesgo reproductivo preconcepcional se puede establecer a partir de la presencia de determinados factores condicionantes que interfieran con el embarazo, parto o puerperio; su magnitud difiere de un caso a otro; por lo que el establecimiento de los mecanismos para que se pueda realizar evaluaciones integrales e individualizadas resulta de gran importancia. ${ }^{(19)}$ 
La atención preconcepcional constituye un conjunto de acciones de Salud dirigidas a la promoción, prevención, valoración e intervención (de ser necesaria esta última) de los diferentes factores predisponentes al respecto. Estas actividades pueden realizarse en el hombre y la mujer de manera individualizada o de conjunto en la pareja, según las características de cada caso. ${ }^{(39)}$

En relación con la importancia de la consulta preconcepcional, Robles ${ }^{(40)}$ plantea que las parejas deben estar preparadas para el embarazo y los hijos por venir sean saludables. Teniendo en cuenta ese criterio, los futuros padres deberían recibir educación y orientación por un facultativo, el que realizará un diagnóstico clínico y psicosocial para identificar los potenciales factores de riesgo ante una gestación, parto y el consecuente bebé.

Ese tipo de consulta se hace bajo los preceptos de los derechos sexuales y reproductivos; con vista al alcance de su función principal que es el adecuado control de la mujer en edad fértil, en cuanto a la presencia de determinadas enfermedades; las que pudieran constituirse en fuente de complicaciones con diferente grado de complejidad. ${ }^{(22)}$

Una vez que la pareja se acerca a la consulta preconcepcional, se desarrollarán las acciones encaminadas a detectar factores de riesgos y patologías asociadas con la gestación; consecuentemente, el médico y la enfermera que brindan atención deben dar orientaciones educativas para prevenir complicaciones durante el embarazo; las que abordarán estilos de vida, seguimiento con el facultativo, prevención de enfermedades, entre otras. ${ }^{(41)}$

Con respecto a las medidas de prevención relativas a la mortalidad materna e infantil, Rivera Miranda et al. ${ }^{(42)}$ sugieren las siguientes para el nivel primario de atención:

- Detección oportuna y conducta adecuada en presencia de riesgo preconcepcional.

- Recoger un historial detallado de la gestación.

- Atención diferenciada e integral ante embarazos de riesgo.

- Remisión de gestantes alto riesgo a instituciones de salud de segundo o tercer nivel.

- Educación para las buenas prácticas nutricionales.

- Realizar control de las infecciones cérvico-vaginales y de vías urinarias.

\section{CONCLUSIONES}

Actualmente, el embarazo en la adolescencia representa un problema de salud pública a nivel mundial, debido a que son considerados de alto riesgo para el binomio madre-hijo.

El primer nivel de salud debe ser el encargado de ejecutar las políticas de gestión del riesgo preconcepcional. La instrumentación de adecuadas medidas de prevención y control garantizarán el éxito en la reducción de los indicadores que involucran al embarazo, el parto y el puerperio; entre las que destacan las referentes a la educación de la población.

Conflicto de intereses: los autores declaran que no existen.

Declaración de contribuciones: María Isabel García Hermida y Geovanna Paola Lucero Arcos 
realizaron recopilación bibliográfica, procesaron e interpretaron la información; además de redactar el artículo.

\section{REFERENCIAS BIBLIOGRÁFICAS}

1. Arrate-Negret MM, Linares-Despaigne MJ, Cuesta-Navarro AL, Isaac-Rodríguez LM, Molina-Hechavarría V. Caracterización epidemiológica de mujeres con riesgo preconcepcional. MEDISAN [Internet]. 2017 [citado 2016 Ago 16]; 21(2), 150-156. Disponible en: http://www.medigraphic.com/pdfs/medisan/mds-2017/mds172c.pdf.

2. Lugones-Botell M. Atención preconcepcional como estrategia básica para prevenir y disminuir la mortalidad materno-infantil. Rev Cubana Obstet Ginecol [Internet]. 2016 Mar [citado 2018 Jun 02]; 42(1). Disponible en: http://scielo.sld.cu/scielo.php?script=sci_arttext\&pi$\mathrm{d}=\mathrm{S} 0138-600 \mathrm{X} 2016000100001 \& \operatorname{lng}=$ es.

3. Díaz-Campos MO, Linares-Segovia B, Osorio-Suarez CE, Álvarez-Jiménez V. Análisis de la morbilidad materna extrema (near miss) en un hospital materno infantil un estudio de casos y controles. Verano Inv Cient [Internet]. 2017 [citado 2018 Jul 21]; 3(2): 381-384. Disponible en : h t t p :// ww w.jovenesen laciencia.ugto.mx/index.php/jovenesenlaciencia/article/view/1740/1244.

4. Holness, N. A global perspective on adolescent pregnancy. Int Journal Nurs Practice [Internet]. 2015 [citado 2018 Jul 21]; 21(5): 677-681. Disponible en: https://onlinelibrary.wiley.com/doi/epdf/10.1111/ijn.12278. https://doi.org/10.1111/ijn.12278.

5. Mejia CR, Delgado M, Mostto F, Torres R, Verastegui-Díaz A, Cárdenas MM, Almanza-Mio C. Maltrato durante el embarazo adolescente: Un estudio descriptivo en gestantes que se atienden en un hospital público de Lima. Rev chil obstet Ginecol [Internet]. 2018 Feb [citado 2018 Jul 12]; 83(1): 15-21. Disponible en: https://scielo.conicyt.cl/scielo.php?script=sci_arttext\&pi$\mathrm{d}=\mathrm{S} 0717-75262018000100015 \& \operatorname{lng}=$ en.

http://dx.doi.or$\mathrm{g} / 10.4067 / \mathrm{s} 0717-75262018000100015$.

6. Flores-Valencia ME, Nava-Chapa G, Arenas-Monreal L. Embarazo en la adolescencia en una región de México: un problema de Salud Pública. Rev. salud pública [Internet]. 2017 Jun [citado 2018 Jun 20]; 19(3): 374-378. Disponible en: http://www.scielo.org.co/scielo.php?script=sci_arttext\&pid=S0124-00642017000300374\&lng=es. $\quad$ http://dx.doi.org/10.15446/rsap.v19n3.43903.

7. Jiménez-González A, Granados-Cosme JA, Rosales-Flores RA. (2017). Embarazo en adolescentes de una comunidad rural de alta marginalidad. Un estudio mixto de caso. Salud púb Méx [Internet]. 2017 [citado 2018 Jun 19]; 59(01): 11-18. Disponible en: http://www.saludpublica.mx/index.php/spm/article/view/8410. DOI: https://doi.org/10.21149/8410. 
8. Mazuera-Arias R, Trejos-Herrera AM, Reyes-Ruiz L. Percepción del embarazo adolescente en el Departamento Norte de Santander, Colombia. Rev. Salud públ [Internet]. 2017 Dic [citado 2019 Jun 02]; 19(6): 733-738. Disponible en: http://www.scielo.org.co/scielo.php?script=sci_arttext\&pid=S0124-00642017000600733\&lng=es.

http://dx.doi.org/10.15446/rsap.v19n6.57679.

9. Monterrosa-Castro Á, Arteta-Acosta C, Ulloque-Caamaño L. Violencia doméstica en adolescentes embarazadas: caracterización de la pareja y prevalencia de las formas de expresión. Iatreia [Internet]. 2017 Mar [citado 2018 Jun 16]; 30(1): 34-46. Disponible en: http://www.scielo.org.co/scielo.php?script=sci_arttext\&pid=S0121-07932017000100034\&lng=en. http://dx.doi.org/10.17533/udea.iatreia.v30n1a03.

10. Zambrano-Quinde OR, Palaú-Guillen MG, Vera-Chiluiza C, Villamar-Oviedo S, Benítez-Chávez AM. (2017). Más allá del uniforme: Una mirada del embarazo adolescente en la ciudad de Manta - Ecuador. Polo del Conocimiento [Internet]. 2017 [citado 2018 Jun 18]; 2(11): 70-84. Disponible en: https://polodelconocimiento.com/ojs/index.php/es/article/view/393/pdf. https://doi.org/10.23857/pocaip.

11. Bulgach V, Zunana C, Califano P, Rodríguez MS, Mato R. Madres adolescentes internadas junto con sus hijos en un hospital de alta complejidad: diferencias entre la adolescencia temprana-media y tardía. Arch argent pediatr [Internet]. 2018 Abr [citado 2018 Jul 23]; 116(2): 160-164. Disponible en: http://www.scielo.org.ar/scielo.php?script=sci_arttext\&pi$\mathrm{d}=$ S0325-00752018000200041\&lng=es. http://dx.doi.org/10.5546/aap.2018.160.

12. Instituto Nacional de Estadística y Geografía. Encuesta Nacional de la Dinámica Demográfica. Prinicipales resultados de encuesta 2014 [Internet]. México: Inegi; 2014. Disponible en: ht t p :// ww w.inegi.org. mx/est/contenidos/proyectos/encuestas/hogares/especiales/enadid/enadid2014/doc/resultados_enadid14.pdf.

13. Departamento Administrativo Nacional de Estadística. (2015). Nacimientos por área de ocurrencia y sexo, según grupos de edad de la madre, total nacional. Cifras del periodo 16 de mayo-30 de junio 2015. Bogotá: DANE.

14. Instituto Colombiano de Bienestar Familiar. Embarazo en Adolescentes: generalidades y percepciones. Bogotá: Observatorio del Bienestar de la Niñez; 2015.

15. Organización Panamericana de la Salud. Indicadores de salud. Aspectos conceptuales y operativos. Washington, D.C.: OPS; 2018.

16. Rojas-Riera JM. (2016). Diseño de estrategia de intervención educativa sobre riesgo preconcepcional. Consultorio $\mathrm{N}^{\circ} 28$. Parroquia Pascuales [Tesis de especialidad]. Guayaquil: Universidad Católica de Santiago de Guayaquil; 2017. Disponible en: http://repositorio.ucsg.edu.ec/bitstream/3317/7409/1/T-UCSG-POS-EGM-MFC-21.pdf.

17. Organización Mundial de la Salud. Salud para los adolescentes del mundo. Una segunda oportunidad en la segunda década [Internet]. Organización Mundial de la Salud: Ginebra; 2014. Disponible en: http://apps.who.int/adolescent/second-decade/files/WHO_FWC_MCA_14.05_spa.pdf. 
18. González R. Chile, un buen lugar donde nacer: Morbimortalidad materna e infantil a nivel global y nacional. Rev Méd Clín Las Condes [Internet]. 2014 [citado 2018 Jul 26]; 25(6): 874-878. Disponible en: https://www.elsevier.es/es-revista-revista-medica-clinica-las-condes-202-pdf-S0716864014706339. https://doi.org/10.1016/S0716-8640(14)70633-9.

19. Hierrezuelo-Rojas N, Alvarez-Cortés JT, Subert-Salas L, González-Fernández P, Pérez-Hechavarría GA. Calidad del proceso en la ejecución del programa de riesgo reproductivo preconcepcional. MEDISAN [Internet]. 2017 Mayo [citado 2018 Jun 19]; 21(5): 518-526. Disponible en: $\quad$ http://scielo.sld.cu/scielo.php?script=sci_arttext\&pi$\mathrm{d}=\mathrm{S} 1029-30192017000500002 \& \operatorname{lng}=\mathrm{es}$.

20. Mendoza-Tascón LA, Claros-Benítez DI, Peñaranda-Ospina CB. Actividad sexual temprana y embarazo en la adolescencia: estado del arte. Rev chil obstet ginecol [Internet]. 2016 Jun [citado 2018 Jul 08]; 81(3): 243-253. Disponible en: https://scielo.conicyt.cl/scielo.php?scrip$\mathrm{t}=\mathrm{sci}$ arttext\&pid=S0717-75262016000300012\&lng=en.

http://dx.doi.or$\mathrm{g} / 10.4067 / \mathrm{S} 0717-75262016000300012$.

21. Instituto Nacional de Estadística y Censo. Registro Estadístico de Nacidos vivos y Defunciones 2015 [Internet]. Quito: INEC; 2015. Disponible en: http://www.ecuadorencifras.gob.ec/$\begin{array}{llllllllllllllllllllllllll}\mathrm{d} & \mathrm{o} & \mathrm{c} & \mathrm{u} & \mathrm{m} & \mathrm{e} & \mathrm{n} & \mathrm{t} & \mathrm{o} & \mathrm{s} & / & \mathrm{w} & \mathrm{e} & \mathrm{b} & - & \mathrm{i} & \mathrm{n} & \mathrm{e} & \mathrm{c} & / & \mathrm{P} & \mathrm{o} & \mathrm{b} & \mathrm{l} & \mathrm{a} & -\end{array}$ cion_y_Demografia/Nacimientos_Defunciones/2016/Presentacion_Nacimientos_y_Defuncion es_2016.pdf.

22. Nápoles-Méndez D. Principales resultados en el Programa de Atención Materno-Infantil durante el 2017 y nuevas estrategias en Cuba para el 2018. MEDISAN [Internet]. 2018 Feb [citado 2018 Jun 23]; 22(2): 217-219. Disponible en: http://scielo.sld.cu/scielo.php?script=sci_arttext\&pid=S1029-30192018000200015\&lng=es.

23. Ministerio de Salud Pública. Autoridades de salud destacan importancia de la medicina familiar en modelo de atención integral [Internet]. Quito: MSP; 2018. Disponible en: https://www.salud.gob.ec/autoridades-de-salud-destacan-i mportancia-de-la-medicina-familiar-en-modelo-de-atencion-integral.

24. Ministerio de Salud Pública. Sistema de vigilancia epidemiológica [Internet]. Quito: MSP; 2018. Disponible en: https://www.salud.gob.ec/tres-cantones-mas-se-integran-al-medico-del-barrio/.

25. Burneo C, Córdoba A, Gutiérrez M, Ordóñez A. Embarazo adolescente en el marco de la estrategia nacional intersectorial de planificación familiar. Quito: ENIPLA; 2014.

26. López N. ¿Cómo cambia el cerebro un aborto inducido?. Cuadernos de Bioética [Internet]. 2012 [citado 2018 Ago 03]; 23(2): 565-84. Disponible en: http:// aebioetica.org/revistas/2012/23/78/565.pdf.

27. Manion A, Wideman M, Tutlewski A. Breastfeeding attitudes among adolescent mothers attending a nutrition breastfeeding support group. Clinical Nursing Studies [Internet]. 2018 [citado 2018 Ago 11]; 6(1): 28-34. Disponible en: http://www.sciedupress.com/journal/index.php/cns/article/view/11649/7643. https://doi.org/10.5430/cns.v6n1p28. 
28. Bermúdez-Pérez RS, Linares-Segovia B, Serrano-Ramos MG, Álvarez-Jiménez V. Proyecto Saelci-Guanajuato. Exposición al alcohol periconcepcional y salud del recién nacido en el Hospital Materno Infantil de Irapuato. Verano de la Investigación Científica [Internet]. 2016 [citado 2018 Jul 19]; 2(1): 1536-1540. Disponible en: http://ri.ues.edu.sv/16803/1/tesis\%20unida.pdf.

29. Hinojosa-Herrera JI, Villegas-Abril CB. Estado nutricional materno y su relación con el estado nutricional del recién nacido en el Centro de Salud Belenpampa - Cusco, 2016 [Tesis en Opción al Título de Licenciada en Nutrición Humana]. Puno: Universidad Nacional del Altiplano; 2018. Disponible en: http://repositorio.unap.edu.pe/bitstream/handle/UNAP/7170/Hinojosa_Herrera_Jackeline_Izkra.pdf?sequence $=1 \&$ isAllowed $=\mathrm{y}$.

30. Gálvez-Henry F, Rodríguez-Sánchez BA, Lugones-Botell M, Altunaga-Palacio M. Características epidemiológicas del embarazo en la adolescencia. Rev Cubana Obstet Ginecol [Internet]. 2017 Sep [citado 2018 Jul 22]; 43(3): 15-27. Disponible en: http://scielo.sld.cu/scielo.php?script=sci_arttext\&pid=S0138-600X2017000300003\&lng=es.

31. Álvarez-Nieto C, Grande-Gascón ML, Linares-Abad M, Ojeda AC. (2017). Análisis del embarazo adolescente: miradas cualitativas a los casos de Bucaramanga y Jaén. Matronas profesión [Internet]. 2017 [citado 2018 Ago 05]; (2): 51-59. Disponible en: https://dialnet.unirioja.es/servlet/articulo? codigo $=6265041$.

32. Restrepo-Martínez M, Trujillo-Numa L, Restrepo-Bernal D, Torres-de Galvis Y, Sierra G. La negligencia y el abuso sexual en la infancia pueden evitarse buscando impactar positivamente el embarazo adolescente. Rev Colomb Psiq [Internet]. 2017 [citado 2018 Ago 09]; 46(2): 74-81. Disponible en: https://www.elsevier.es/en-revista-revista-colombiana-psiquiatria-english-edition--479-articulo-sexual-abuse-neglect-situations-as-S2530312017300206. https://doi.org/10.1016/j.rcp.2016.05.004.

33. Bravo VP, Contreras MA, Dois CA, Contreras MM, Rojas RA. Necesidades psicosociales y características de una intervención online para apoyar a las madres adolescentes. Rev chilena obstet ginec [Internet]. 2017 [citado 2018 Jul 23]; 82(5): 566-573. Disponible en: http://www.revistasochog.cl/files/pdf/AI_031.pdf. https://dx.doi.org/10.4067/s0717-75262017000500566.

34. Vicente-Peña E, Peraza-Rodríguez G, Sánchez-Nuñez LO. Medicina Interna. Diagnóstico y tratamiento. 2 ed. La Habana: Editorial Ciencias Médicas; 2016.

35. Duque PA, Valencia-Rico CL, Araujo JJ. Factores sociodemográficos y factores de riesgo preconcepcionales en padres y madres de niños con cardiopatías congénitas. Enfermería Clínica [Internet]. 2018 [citado 2018 Jul 17]; (In press). Disponible en: https://www.elsevier.es/en-re$\mathrm{v}$ i s t a - e n f e r m e r i a - c 1 i n i c a - e n g 1 i sh-edition--435-articulo-socio-demographic-preconception-risk-factors-in-S244514791830075 4. DOI: https://doi.org/10.1016/j.enfcli.2018.03.003.

36. Lugones-Botell M. La mortalidad materna, un problema a solucionar también desde la Atención Primaria de Salud. Rev Cubana Med Gen Integr [Internet]. 2013 Mar [citado 2018 Jun 27]; 29(1): 1-2. Disponible en: http://scielo.sld.cu/scielo.php?script=sci_arttext\&pi$\mathrm{d}=\mathrm{S} 0864-21252013000100001 \& \operatorname{lng}=\mathrm{es}$. 
37. García-Odio A, Izaguirre-Mayor DR, Álvarez-Bolivar D. Impacto de la anemia para una embarazada e importancia del riesgo preconcepcional. Rev Cubana Med Gen Integr [Internet]. 2017 Mar [citado 2018 Jul 14]; 33(1): 146-153. Disponible en: http://scielo.sld.cu/scielo.php?script=sci_arttext\&pid=S0864-21252017000100013\&lng=es.

38. Vigil-Iglesias AA, Rodríguez-González LM, Villatoro-Godoy KG, Méndez-Flamenco JRA. Factores de riesgo participantes en las morbilidades obstétricas de embarazadas del municipio nueva Concepción, Chalatenango, marzo - junio de 2017 [Tesis en Opción al Título de Doctor en Medicina]. San salvador: Universidad de El Salvador; 2017. Disponible en: http://ri.ues.edu.sv/16803/1/tesis\%20unida.pdf.

39. Alvino-Mamani JL, Rojas-Espinoza CH. Factores de riesgo en la etapa preconcepcional en usuarias de los consultorios de planificación familiar del Instituto Nacional Materno Perinatal [Tesis en opción al grado de Licenciada en Obstetricia]. Lima: Universidad Mayor de San Marcos; 2015. Disponible en: http://cybertesis.unmsm.edu.pe/xmlui/bitstream/handle/cybertesis/4627/Alvino_mj.pdf? sequence=1\&isAllowed=y.

40. Robles E. Motivos para asistir a la consulta preconcepcional. Hospital Enrique C. Sotomayor. Quito: HECS; 2013.

41. Cruz Hernández J. Segundo Consenso Cubano de Diabetes y Embarazo. Rev Cubana Endocrin [Internet]. 2018 [citado 2018 Ago 07]; 29(1): 1-29. Disponible en: http://www.revendocrinologia.sld.cu/index.php/endocrinologia/article/view/97/87.

42 Rivera-Miranda MA, Nadia-Teresa LL, Baró-Bouly T. Asfixia al nacer: factores de riesgo materno y su repercusión en la mortalidad neonatal. Rev Inform cient [Internet]. 2017 [citado 2018 Jul 23]; 96(6): 1143-1152. Disponible en: https://dialnet.unirioja.es/descarga/articu1o/6166376.pdf.

Recibido: 13 de octubre de 2018

Aprobado: 19 de noviembre de 2018 